\title{
Establishment of the Subtitle on Materials for Evaluating Intellectual Ownership
}

\author{
Kyu-Tae Lee ${ }^{1}$, Hyun-Chang Lee ${ }^{2}$ and Jang-Geun $\mathrm{Ki}^{3}$ \\ ${ }^{1,2}$ Div. of Information \& Communications, Kongju National University, Korea \\ ${ }^{3}$ Dept. of Electrical and Electronic and Control Engineering, Kongju National \\ University, Korea \\ ${ }^{1}$ ktlee@kongju.ac.kr, ${ }^{2}$ hclee@kongju.ac.kr, ${ }^{3}$ kjg@kongju.ac.kr
}

\begin{abstract}
On increasing the illegal copyright of ICT(Information Communication Technology) devices, there was issued to conflict on the Intellectual property. A copyright evaluation process is a way of resolving an illegal modification between the original device and the suspected device both on software and hardware. The compared result is extracted from the evaluation of each subtitle item with detail to seeking objective result. Therefore, each subtitle evaluated item have a serious concentration as to it has a sensitive value in decision. In this paper, it was analyzed three cases of practical evaluation work such as software, hardware and printed board issues. Also we suggested the procedure for selecting the detailed item as for the actual evaluation materials.
\end{abstract}

Keywords: Copyright, Illegal copy, evaluation. Source code, comparison tool, accuser, defender

\section{Introduction}

Usage of the digital devices is now expanded and increased with its easy usability, producing and distribution on every public area. Therefore it has a difficulty to protect to an illegal copy and transformation on copyright owner. And there are many issues in copyright protection to keep the original ownership of contents developer from lots of attacks. Also it can be take down the creative activity of contents designer and even developing to criminal case as to obstructing new contents product. By these reason, copyright protection is becoming important issues to keep and growing up creative contents of original producer against illegal activities. In the domestic organization, the copyright association, is now taking a charge into copyright protect and contents verification by comparison to the suspected materials, when there is conflict issues in copyright ownership. The processing of comparison is a way to extract a numerical likelihood throughout matching process between original and suspect contents. [1]

The evaluation to the requested subtitle item is different according to the type of conflict. But in case of assigning the subtitle item, evaluation experts need to reassign a detail subtitle so as to high quality technique on each development steps. These subtitle items should be extracted from the object materials and knowledge of the expert on the processing. The expert should have an objective knowledge to the evaluation materials and explain a reason how these subtitle could be assigned for this comparison. On the case of assigning a weight factor to each subtitle, expert use his subjective knowledge of the technique to the copyright.

In this study, we verified and suggested to selecting the detailed item as for the actual evaluation materials. Also it shows a way to adapt a reasonable comparison process and extract a likelihood of both contents.

Received (June 5, 2017), Review Result (August 6, 2017), Accepted (September 15, 2017) 


\section{Evaluation of Object Material}

The procedure of searching similarity from object materials is shown on Figure 1. for the ICT devices and program source. At first, a person who has disadvantages on his properties accuses a person who did an illegal copy to investigatory agency. The agency begins to investigate the materials submitted from both sides. Then after ask an expert opinion such as copyright association. In this step, if it can be pointed out a specific part to investigate, it is possible to assign specific search items to copyright association with agreement. The evaluation expert takes an investigation for the similarity to the specific item or total items as requested.

For the evaluation task, assigning a subtitle item is important for reducing an objective result and applying a weight factor to each item. A designation for evaluation experts is assigned by the copyright association for analyzing the subjected materials. Also searching for open source to specific items is performed to find out an originality of the property owner on the materials. [9]

On doing analysis, the decision depends on the evaluation expert. He deduces the original evidence and try to find out which kinds of knowledge used, such as common or specific.

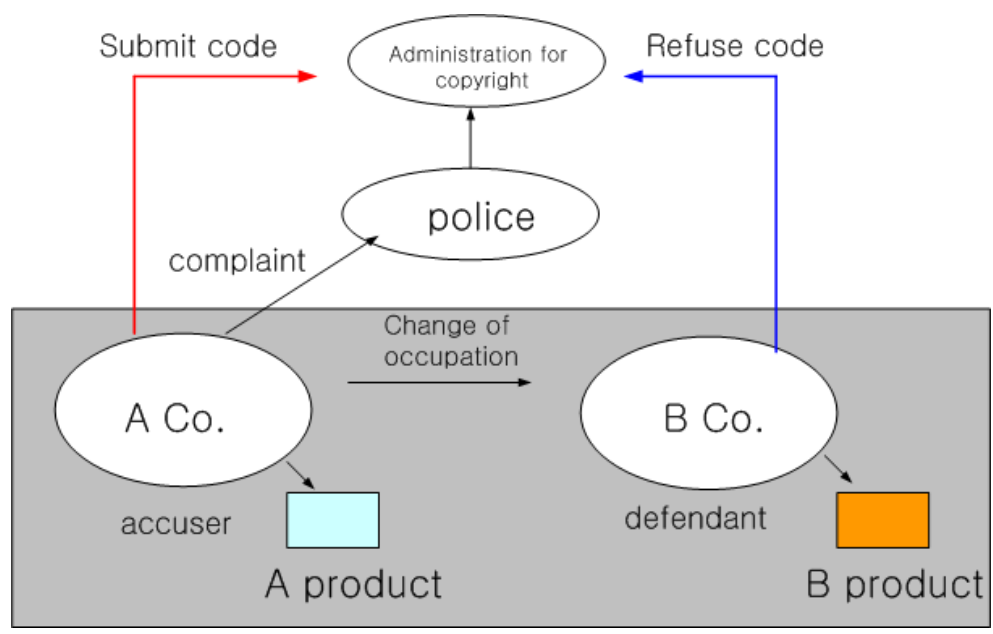

Figure 1. A Flow of Evaluation

On extracting evaluation likelihood values on the object materials, there are two way of an original base and suspect base by each source code guideline. At this moment, there can be excluded to different result values and can make a dispute on court. Figure 2 shows an illustration of original material and partially illegal copy material case.

In this figure, left side is an original code block and right side is an illegal code block. For each blocks, the $\mathrm{X}$ means common part of both original and suspect code, and $\mathrm{Y}$ and $\mathrm{Z}$ means additive code for original program and suspect program. The ' $\mathrm{xx}$ ' means the code block that a suspect developer takes a carbon copy from original code, showing exactly same code lines. As a result we can calculate and count a likelihood value as to Table 1.

The matched code lines are $40 \%$ to original developer but this value is recalculated $30 \%$ to suspected developer in total generated lines. So decision of reference code lines is debatable issues by the evaluation expert. According to the evaluation material, reference code lines can be changed and also even it has low similarity value it can be serious factor to prove an illegal copy event. 


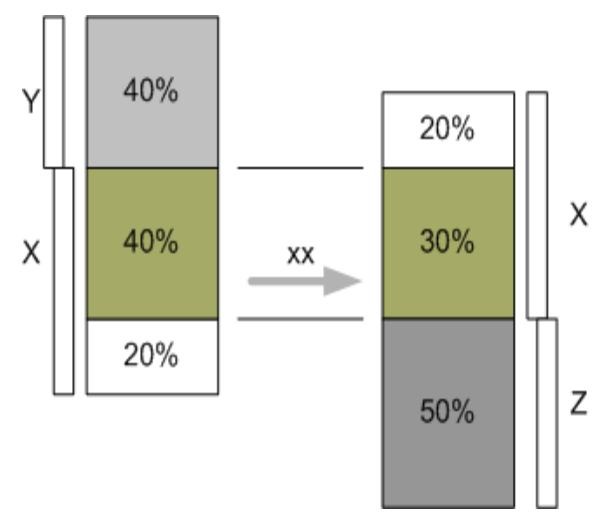

Figure 2. Compare Processing with Materials

After evaluation, original based likelihood value is $40 \%$, and suspect based value is $30 \%$, even with a same material comparison trial. It can be serious on both side to accuser and defender.

Table 1. Evaluation Based Result

\begin{tabular}{|c|c|c|}
\hline method based & equation & $\begin{array}{c}\text { likelihood } \\
(\%)\end{array}$ \\
\hline \multirow{2}{*}{ original } & $\mathrm{xx} /(\mathrm{X}+\mathrm{Y}) * 100$ & \multirow{2}{*}{$40 \%$} \\
\cline { 2 - 2 } & $40 /(60+40) * 100$ & \\
\hline \multirow{2}{*}{ suspect } & $\mathrm{xx} /(\mathrm{X}+\mathrm{Z}) * 100$ & \multirow{2}{*}{$30 \%$} \\
\cline { 2 - 2 } & $30 /(50+50) * 100$ & \\
\hline
\end{tabular}

Generally, illegal program is constructed by modification from original code source cause it has a key technology code block which is hard to make. Also they add a new program source code block to original program which has a creative idea of property owner. Sometimes besides key function block of original code, there are trials to hide illegal acts by modifying easy interface blocks such as GUI or display colors etc. Therefore In these cases, suspect code based evaluation method is not correct and the likelihood value does not have critical meaning to make decision. So basically source code based evaluation is accepted and exceptionally suspect code based method is adapted when original code is included to the suspected code material with too many part. Finally there is no principle which method is better, that is mainly the right to evaluation expert decision under conflict material inspection.

\section{Evaluation Cases}

\subsection{Hardware Material}

The object material of hardware system has a configuration of electrical circuit and processor module and software as shown in Figure 3. This device works to amplify human voice for hearing aid with four blocks drawn. When a voice inputs to microphone, preamplifier circuit increases the signal level and comparing it to feedback signal from amplified output parts. The power amplifier used to drive a physical speaker to speak out loud voice. The feedback block, ALC(Automatic Level Control) is used for output signal to go back to preamplifier block to control output level. Finally, the controller block consist of microprocessor and software program to control key input with useful UI(User Interface) and to indicate operation control such as battery status and operation status and volume control. 


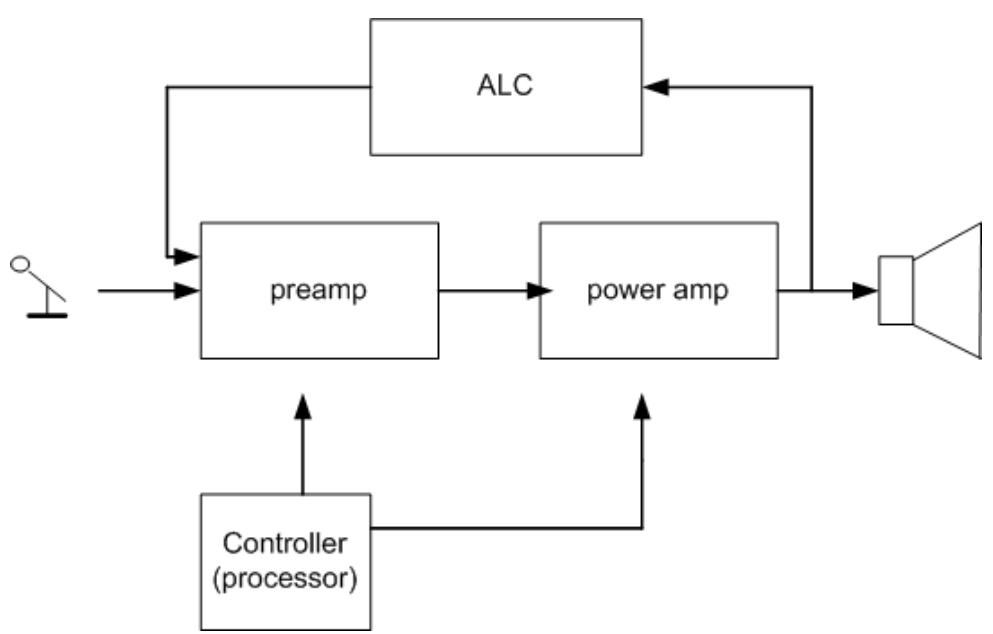

Figure 3. General Hearing Aid Structure

The circuit has an operation to automatic level control to keep proper level for hearing voice. This device has to be designed for attach to human body comfortably. Especially it is installed on the lower part of the ear, so developer drives a special technique which has small size and light weight, sightless structure configuration.

In this device, the core part is ALC block. It works to keep the output level to constant level from analog microphone while input level is going up and down continuously. It also called to ALC or AGC(Automatic Gain Control). Analog circuit of ALC is shown in Figure 4. It works to convert an analog output signal to DC voltage level with two diodes. This DC voltage level works as feedback signal to input signal of base terminal of BJT(Bipolar Junction Transistor).

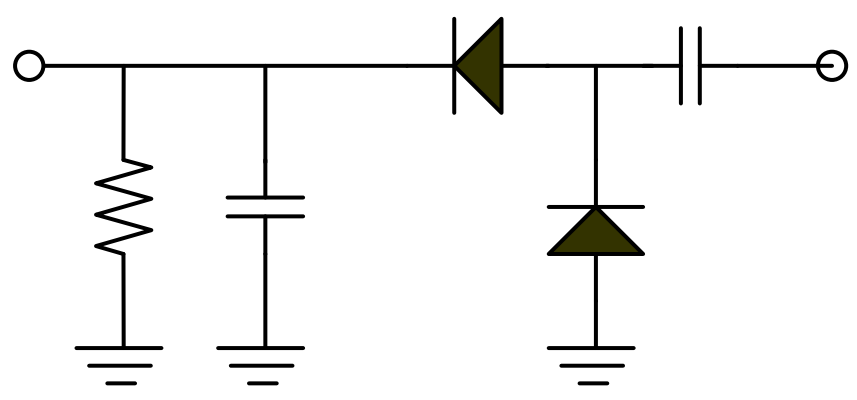

Figure 4. Feedback Circuit

The evaluation material can be classified in 6 subtitles, such as small signal amplifier, ALC circuit, Power amplifier, MCU processor, recharge and LED indicator. This device is designed for hearing aid person such a deaf person or old aged person who needs a voice amplifier device. The core technology of this device is to make to keep a constant voice level to a human ear. So amplifier block to analog signal and feedback to keep level in constant is main blocks and the other things are used for supporting these functions. Small signal amplifier amplifies a signal coming into the ear to drive a speaker attached to end point and this signal is mixed with feedback signal from output to regulate the voice level. ALC circuit part designed to analog components is a kind of an accumulator to convert analog signal to DC voltage level with two diode and capacitor. Power amplifier drives an amplified small signal to physical high current speaker component. MCU processor controls all of parts to keep the system operation in time. This procedure is designed with $\mathrm{C}$ language to interface to input digital signal and output an indication signal to the device panel. This device works with a recharge battery inside system box. The device indicates how long the energy can support as to LED lamp. 
Each subtitle has in charge of strong role in the device. Also it can be classified in 2 categories, component amount and similarity of circuit drawing. Similarity of component amount shows $83.1 \%$ results, therefore it is suspected defender could modify accuser device as a reference.

Table 2. Similarity of Component Amount

\begin{tabular}{|c|c|c|c|c|}
\hline NO. & subtitle & accuser & defendant & simliarity \\
\hline 1 & small signal amplifier & 23 & 22 & 95.6 \\
\hline 2 & ALC circuit & 9 & 9 & 88.8 \\
\hline 3 & Power amplifier & 8 & 7 & 87.5 \\
\hline 4 & microprocessor & 15 & 18 & 26.6 \\
\hline 5 & recharge & 11 & 11 & 100 \\
\hline 6 & LED indicator & 10 & 10 & 100 \\
\hline & & & & $83.10 \%$ \\
\hline
\end{tabular}

\subsection{Software Material}

The evaluation system is about a treadmill for health exercise. It is composed a simple mechanic device and software programmed. The characteristic of system is running belt system with motor and control board included microprocessor. Program works with sensor input signal and display all of information to indicate. Also it has functions of storing and transmitting of health information to the person. A flow of software program is as Figure 5. At first, programmer creates a flowchart of operation sequence. And with proper processor and program language, software codes are generated. After compiling, machine code downloaded to the processor as binary code values. These kinds of device have specific characters in copyright, such as interface logic, software language and operation sequence.

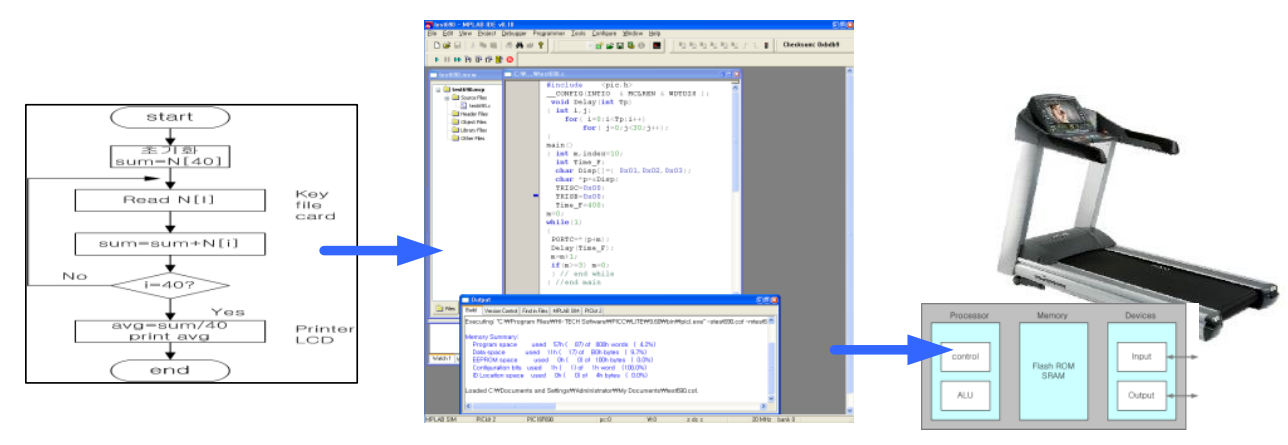

Figure 5. System Development Flow

After analysis of materials, there are extracted to three files of defender and one file of accuser as Table 3. In this paper, file names are assigned to KS for accuser and A, B and $\mathrm{C}$ for defender. All of program code was generated with $\mathrm{C}$ language under same microprocessor. 
Table 3. Extracted Files by Analysis

\begin{tabular}{|c|c|c|c|}
\hline No. & filename(lines) & owner & knicname \\
\hline 1 & main.c(3012) & accuser & KS \\
\hline 2 & main-p9.c(3627) & defender & A \\
\hline 3 & main-p8.c(3787) & defender & B \\
\hline 4 & main-t8.c(3413) & defender & C \\
\hline
\end{tabular}

A number of lines to accuser file show less than lines of defender. It tells us that defender modify files of accuser and add some program code in main function. The source code comparison will be done by the method explained in Figure 2.

There are many commercial tools for comparing file by file such as 'diff', 'Eclipse', 'Ediff', 'Windiff' and etc. In this paper, we use a commercial comparison tool called 'Beyond compare ver.3.' which was released by Scooter software.[8] It supports useful compare functions and convenient works such as, text by text and file by file, and also folders available as shown in Figure 6.

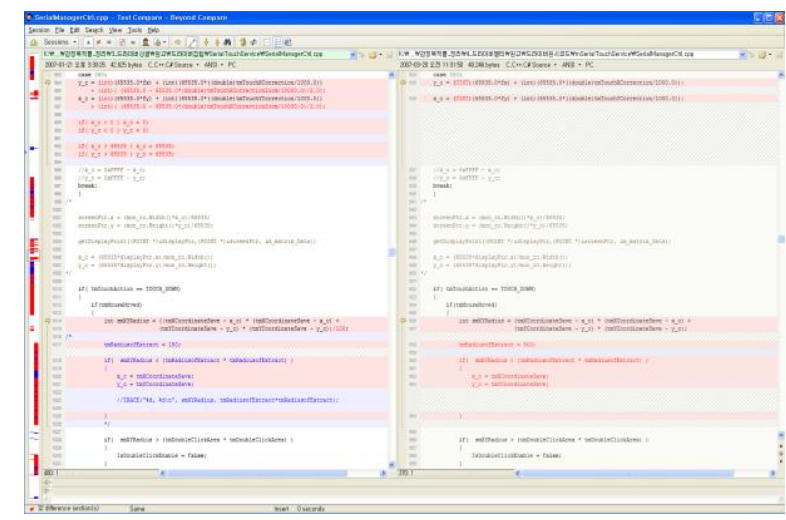

Figure 6. Beyond Compare Result Window

A source code was made with general $\mathrm{C}$ language operated in microprocessor. The materials of main source code work in treadmill health system as background processing which means endless looping code. There are common usages with 'while' function of code in which all function of devices is listed and selected by input command. In the 'while' function, 'case' command is used to select the functions, so listed functions has a originality of developer for keeping the copyright. Evaluation task will be processed by comparing the all functions in 'while' command.

Evaluation is started with verification of whether it has open source or not in the program code. If there are some open sources in public usage, it excludes all of public source code before doing comparison to the material files. In this experiment, there are found to 9 code block as a subtitle in the 'while' function inside.

1) Predefine module

This code block starts with '\#define'. There are 125 code lines in accuser and defender code and after comparison, 60 code lines are matched as the same code with $48 \%$ similarity. Also some of comment block was found as the same code.

2) Variable define module

In the program source code, variable is used as a temporary storage which can be changed the value while execute program. In this module, there are 87 lines matched as the same code among 200 lines of defender and accuser source code. Similarity value is 
43.5\%. But this part was used as common definition value so there is no specific copyright meaning.

3) Communication Interrupt module

This block works for defining data communication type when interrupt occur for serial data transfer. There are 25 lines matched as the same lines in both source code blocks. Similarity shows $100 \%$ matching result.

4) Timer Interrupt module

The Timer included in microprocessor make a periodic impulse as an interrupt signal to work special designated task. There are 86 lines matched as a same code among the 203 lines in both codes. The similarity value has $42.3 \%$ as a copyright to developer.

5) Display function module

The display function works as an information output to system user on the display panel. There are 86 lines matched as a same code among the 397 lines in both codes. The similarity value has $21.6 \%$.

6) Time/distance function module

The function works as a storage which it calculate exercise time, distance, calorie consumption. There are 122 lines matched as a same code among the 139 lines in both codes. The similarity value has $87.7 \%$.

7) Health start/stop function module

This part takes input data from sensors, such as exercise start time, stop time and heart rate. There are 38 lines matched as a same code among the 95 lines in both codes. The similarity value has $40.0 \%$.

8) Main initialization module

This module works initialization of main function and input push button of start button and stop button. There are 21 lines matched as a same code among the 60 lines in both codes. The similarity value has $35.0 \%$.

9) Main inc/dec setting module

This module works to change speed on treadmill when button pressed for increase or decrease with plus and minus button. There are 72 lines matched as a same code among the 158 lines in both codes. The similarity value has $45.5 \%$.

Finally, total similarity result shows a matched value, $51.1 \%$ as shown in Table 4 . By this data, the source code is suspected of being modification of accuser code as a reference.

Table 4. Similarity Result of 'Main' Function

\begin{tabular}{|c|c|c|}
\hline & subitems & similarity(\%) \\
\hline 1 & predefine & 48 \\
\hline 2 & variable define & 43.5 \\
\hline 3 & com. Interrupt & 100 \\
\hline 4 & timer interrupt & 42.3 \\
\hline 5 & dsiplay func. & 21.6 \\
\hline 6 & time, distance func. & 87.7 \\
\hline 7 & health start/stop & 40 \\
\hline 8 & main init & 35 \\
\hline 9 & main inc/dec set & 45.5 \\
\hline & & 51.51 \\
\hline
\end{tabular}




\subsection{PCB Material}

The development work of Hardware system is completed by PCB(Printed Circuit Board) assembly. This board is designed to fix a device size, component position, location of terminals such as power, input, output and key pad. The design of the board is started with block diagram and circuit diagram of the system as shown Figure 7. There are many kinds of PCB design tools released in commercial market. The tools are depended on the developer who is used to be to work such as ORCAD, PADS. But the last file designed is Gerber file to share with all of designer.

PCB is a practical circuit board which is composed of printed lines to connect a all of component terminal and let us know the location of components. This has an originality of developer knowledge in design. That knowledge is contained in PCB size, types of pattern, component location, components distance, connection line and width of pattern. All of these items can be a copyright of owner. Therefore PCB is mainly dependent on the designer. Also the board is very sensitive in high frequency as an interference called EMI(Electro Magnetic Interference). In case of designing DDR SDRAM(Double data rate synchronous dynamic random access memory), it works on fast data transition rate on $400 \mathrm{MHz}$ frequency. To make a line between CPU and DDR is needed a damping resistor to decrease noise and match for impedance.

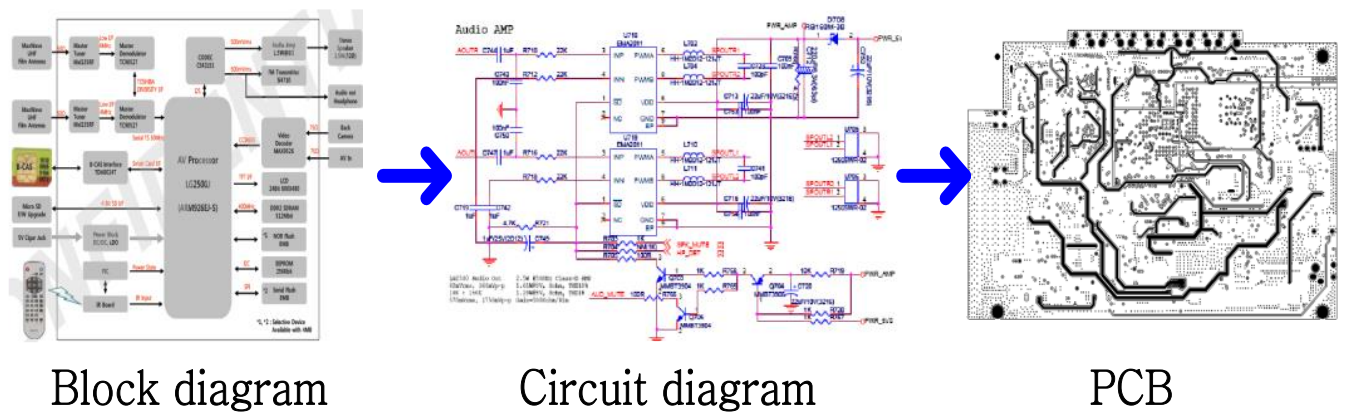

Figure 7. Flow of PCB Design

Generally, PCB design is requested to commercial expert company to make Gerber files. In this case the board was composed to 6 layers and two silk screen layers. Silk screen layer is used to inform all of component location, direction, and values by printing down to the PCB board on surface. One is used for top of board and the other is printed on bottom of the board. The location, width and length of connection pattern is important information for proving copyright of owner. Comparison of PCB board is done by verify each 8 layer pattern with insights of expert.

Evaluation was compared with each 8 layers to verify a similarity of the board. All of layer was found to be mismatched as shown Table 5. This means even though circuit diagram would be same, board design could be totally different product. 
Table 5. Similarity of Each PCB Layers

\begin{tabular}{|c|c|c|}
\hline No. & PCB layer & similarity \\
\hline 1 & top side(top) & mismatch \\
\hline 2 & plane2 GND & mismatch \\
\hline 3 & plane3(inner layer3) & mismatch \\
\hline 4 & lane4 (inner layer4) & mismatch \\
\hline 5 & layer5 VCC & mismatch \\
\hline 6 & Bottom side & mismatch \\
\hline 7 & silk screen top & mismatch \\
\hline 8 & silk screen bottom & mismatch \\
\hline
\end{tabular}

\section{Conclusion}

The evaluation of the object material has two type of request. One is a case of designated request items to similarity verification and other one is a request to verify all of copyright available overall. Evaluation item is important factor to make a decision for possibility of illegal copy. Even if there are designated request item, expert should trace to the design processing how the device was designed and produced and assembled. And it must be tried to verify what sort of technology was used, whether it has originality or public knowledge. So Evaluation expert has to consider technically detail items than general or requested one. The detail items were assigned by expert and device construction skill. The expert can assign a weight factor to each item by his special knowledge to extract fair similarity value.

Also there are two comparison methods to extrude numeric values for similarity, these are comparison to original object based and suspected object based. These results have different values even to the same materials. Therefore the average of these two results could be accepted for the objective impartial decision.

In this paper, we analyze three types of the practical evaluation process and show how to define and assign the detail items to get reasonable similarity value. Also we show an objective evaluation guideline through a case study. Evaluation method and comparison method could be selected and decided by the knowledge of expert.

\section{Acknowledgments}

This paper is a revised and expanded version of a paper entitled [A Comparison Method of Intellectual Ownership materials] presented at [Kyutae Lee, ICGHIT 2017 Hangzhou China, February 2017].

\section{References}

[1] Korea Software Copyright Committee," Establishment of the mid-and long term strategies to protect original technology of software", (2009).

[2] R. K. Gupta, "Introduction to Embedded system", ICS 212, winter, (2002).

[3] Embedded process system, Hanback electronics, (2003).

[4] Getting started with HBE-EMPOS II, Hanback electronics, (2004).

[5] Intel PXA255 Processor Developer's Manual

[6] Joseph Yiu, ARM Cortex-M3 guide, ITC, (2011).

[7] www.copyright.or.kr

[8] www.scootersoftware.com

[9] K. Lee and S. Kim, "Source Code Verification on Embedded Control Devices," International Journal of Software Engineering and Its Applications, vol. 10, no. 5, (2016), pp. 69-76.

[10] K. Lee, "Classification for Similarity Evaluation on Embedded System", The journal of the Korea Software Assessment and Valuation Society, vol. 11, no. 1, (2015), pp.47-51. 
[11] K. T. Lee, W. Ye, H. M. Cho, D. H. Kim and H. Ahn, "A Comparison Method of Intellectual Ownership materials”, ICGHIT 2017 conference, Hangzhou China, (2017), pp. 84-85,.
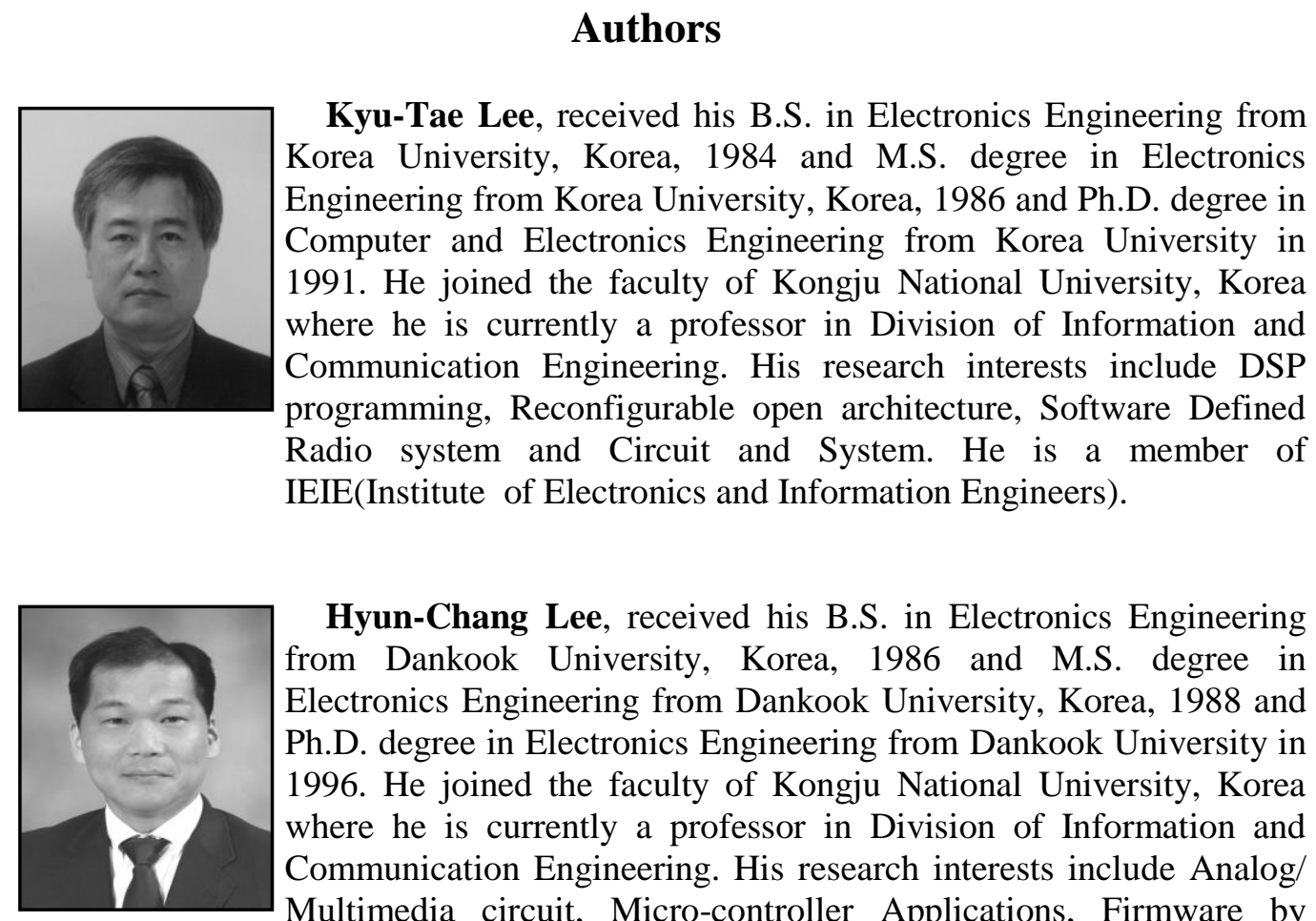

Hyun-Chang Lee, received his B.S. in Electronics Engineering from Dankook University, Korea, 1986 and M.S. degree in Electronics Engineering from Dankook University, Korea, 1988 and $\mathrm{Ph} . \mathrm{D}$. degree in Electronics Engineering from Dankook University in 1996. He joined the faculty of Kongju National University, Korea where he is currently a professor in Division of Information and Communication Engineering. His research interests include Analog/ Multimedia circuit, Micro-controller Applications, Firmware by assembly language and Circuit for Mechatronics.

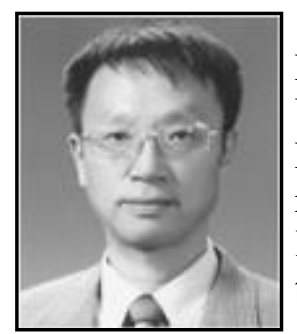

Jang-Geun Ki, is currently a professor in the Department of Electrical \& Electronic \& Control Engineering, Kongju National University in South KOREA. He received B.S., and M.S. degrees in Electronics from Korea University, South KOREA, in 1986 and 1988, respectively. In 1992, he received Ph.D. degree of Electronics at Korea University. His main research interests include telecommunication protocols, mobile networks and Future Internet. 